\title{
Streptococcal serotypes newly associated with epidemic post-streptococcal acute glomerulonephritis
}

\author{
H. F. M. REID, D. C. J. BASSETT*, EVA GAWORZEWSKAt, G. COLMAN $†$ and T. POON-KING
}

Trinidad and Tobago Public Health Laboratory, PO Box 164, Port of Spain, Trinidad, West Indies, "PAHO/WHO Caribbean Epidemiology Centre, Port of Spain, Trinidad, tCentral Public Health Laboratory, Colindale, UK, and $\ddagger$ General Hospital, San Fernando, Trinidad

\begin{abstract}
Summary. The increasing incidence of reported scabies in Trinidad, from 24.2/ 100000 population in 1984 to $59 \cdot 5 / 100000$ in 1985 , led to a careful monitoring in 1986 of all patients with post-streptococcal acute glomerulonephritis (PSAGN). There were 181 cases of PSAGN; $84 \beta$-haemolytic streptococcal isolates from 72 patients were sent to the Central Public Health Laboratory, Colindale, for grouping and serotyping. The PSAGN epidemic of 1986 was bimodal. Streptococci of M-type 73 appeared to be associated with the first phase (March-May) and comprised $20 \%$ of the isolates serotyped. New to Trinidad, streptococci of M-type 48 (4\% of the isolates serotyped) preceded the first phase of the epidemic and were isolated from two patients with PSAGN. Provisional type (PT) 5757, also new to Trinidad, had been previously identified only among serotypes from the United Kingdom and the Federal Republic of Germany. This type was isolated from seven patients, in six from skin lesions and in one from the throat. PT 5757 occurred during the first wave of the epidemic and comprised $14 \%$ of the strains serotyped. Thus, the first wave of the bimodal epidemic involved serotypes M73, M48 and PT 5757. The more intense second phase (July-October) was associated with the previously documented nephritogenic M-type 55.
\end{abstract}

\section{Introduction}

Post-streptococcal acute glomerulonephritis (PSAGN) is endemic in Trinidad. Streptococcal infections of the skin and of the throat are common, yet only a small percentage of the population develop renal damage with immune complex deposition. Since 1952 there have been at least six periods of epidemic PSAGN in South Trinidad ${ }^{1,2}$ the most widespread of which occurred in 1971 when 745 cases were admitted to the General Hospital, San Fernando; many of these cases follow secondarily infected scabietic lesions. ${ }^{3} \beta$-Haemolytic group-A streptococci of various M-types (49, $55,57,60$ ) have been associated with outbreaks of the disease ${ }^{2}$ although, recently, cases of PSAGN associated with group-G streptococci have been reported. ${ }^{4,5}$ The increase in the incidence of reported scabies from $24 \cdot 2 / 100000$ population in 1984 to $59 \cdot 5 / 100000$ in $1985^{6}$ led to a careful monitoring in 1986 of all PSAGN patients admitted to the General Hospital, San Fernando.

Received 14 Sep. 1989; accepted 13 Nov. 1989
This paper reports evidence of nephritogenicity in additional streptococcal serotypes isolated during the PSAGN outbreaks of 1986.

\section{Materials and methods}

\section{Patients}

The PSAGN patients investigated were in-patients of the General Hospital, San Fernando, admitted during 1986. In each case, the diagnosis was established clinically by one of us (T.P-K.) as previously described ${ }^{7}$ i.e., by the presence of various combinations of the following: oedema, haematuria, proteinuria, pyoderma, sore throat and signs of encephalopathy, reduced serum complement $\mathrm{C} 3$, and raised anti-streptolysin $\mathrm{O}$ titre or anti-hyaluronidase titre. Patients were admitted to the study only when both laboratory and clinical evidence were consistent with a diagnosis of PSAGN. The ages of the patients were from 2 to 35 years.

\section{Streptococcal isolates}

From each PSAGN patient admitted to the Hospital, throat swabs and swabs from skin lesions, if present, were collected and cultured on blood agar. Isolates of $\beta$ haemolytic streptococci were then sent to the Central 
Public Health Laboratory, Colindale, United Kingdom, for grouping and serotyping. A combined T- and $\mathrm{M}$ typing scheme was supplemented with detection of opacity factor (OF). ${ }^{8}$ The T-types were determined by the agglutination of trypsinised cell suspensions and $\mathrm{M}$ antigens were detected in Lancefield acid extracts by double diffusion tests in agarose gel.

\section{Results}

\section{Isolates from PSAGN patients}

Amongst the 181 patients with PSAGN, $84 \beta$ haemolytic streptococci isolated from the throats and skin of 72 patients were sent to the Central Public Health Laboratory. Only 52 (61.9\%) of these isolates belonged to group A and were successfully grouped and typed. The other 32 strains could not be typed for various reasons; $13(15.24 \%)$ lost viability in transit to the UK., $7(8.3 \%)$ belonged to group B, $1(1.2 \%)$ was an $\alpha$-haemolytic streptococcus, and $11(13.1 \%)$ failed to group with either group A or B antisera.

\section{Streptococcal serotypes}

The 52 group-A strains successfully serotyped comprised $19(36.5 \%)$ M-type 55, $11(21 \cdot 2 \%)$ Mtype $73,7(13.5 \%)$ PT5757, $4(7 \cdot 7 \%)$, M-type 11,4 $(7 \cdot 7 \%)$ PT $180,3(5.8 \%)$ M-type 48 and $4(7.7 \%)$ that were non-typable. The table shows the presenting symptoms and other relevant clinical data on PSAGN patients whose streptococcal isolates were serotyped.

$M$-type $55(T 55 / O F-)$. The 19 isolates of this type were from 18 patients, 16 of whom were female, aged 2-30 years. Fourteen of the 19 isolates were from 13 patients who lived in County Victoria, two from Caroni, two from St Patrick and one from Nariva/Mayaro. There were seven isolates from skin lesions and 12 from throat cultures, one patient yielding isolates both from the skin and from the throat.

$M$-type $73(T 3 / 13 / B 3264 / O F+)$. There were 11 such isolates from 10 patients, all children (aged 410 years). Five isolates were from skin lesions and six from throat swabs; one patient yielding both skin and throat isolates. There were six female and four male patients; nine were from County Victoria, one from Caroni and one from St Patrick.

$M$-type PT 5757 (T5/27/44/OF-). These were from seven patients, three male and four female, aged 2-16 years. Six of the isolates were from skin lesions and one from the throat. Four patients were from County Victoria, two from St Patrick (including the one that gave a throat isolate) and one from Caroni.

$M$-type $48(T 4(28) / O F+)$. This strain was isolated for the first time in Trinidad. There were three isolates from two patients - a 3-year-old male (throat and skin) and a 13-year-old female (throat), both from County Victoria.

$M$-type PT $180(T 5 / 27 / 44 / O F+)$. This strain is uncommon in Trinidad. Two male and two female patients, aged 10-24 years, yielded four isolates, two from the throat and two from the skin. Two patients lived in County Victoria, one in Caroni and one in St Patrick.

$M$-type $11(T 11 / O F+)$. There were four isolates from two females and one male aged $4-8$ years, all

Table. Clinical and laboratory data on PSAGN patients with different streptococcal serotypes

\begin{tabular}{|c|c|c|c|c|c|c|c|c|c|c|}
\hline \multirow{2}{*}{$\begin{array}{l}\text { Serotype } \\
\text { (number of } \\
\text { patients) }\end{array}$} & \multicolumn{10}{|c|}{ Number of patients with clinical finding of } \\
\hline & $\mathrm{Oe}$ & Haem & Prot & ST & SL & $\mathbf{F}$ & $\uparrow \mathbf{B P}$ & $\mathrm{TC}+\mathrm{ve}$ & SS + ve & $\downarrow \mathrm{C} 3$ \\
\hline M 55(18) & 18 & 18 & 18 & 11 & 14 & 9 & 13 & 12 & 7 & 18 \\
\hline M $73 *(10)$ & 9 & 9 & 10 & 5 & 7 & 5 & 7 & 5 & 5 & 10 \\
\hline PT $5757^{*}(7)$ & 7 & 7 & 7 & 2 & 7 & 4 & 6 & 1 & 6 & 7 \\
\hline M 48*(2) & 2 & 2 & 2 & 1 & 2 & 1 & 2 & 2 & 1 & 2 \\
\hline PT 180(4) & 3 & 3 & 3 & 2 & 3 & 1 & 3 & 1 & 2 & 4 \\
\hline$M 11(3)$ & 3 & 3 & 3 & 1 & 3 & 0 & 1 & 3 & 1 & 3 \\
\hline Non typable (3) & 3 & 2 & 3 & 1 & 3 & 1 & 3 & 1 & 2 & 3 \\
\hline
\end{tabular}

Oe, Oedema; Haem, haematuria; Prot, proteinuria; ST, sore throat; SL, skin lesions; F, fever; $\uparrow B P$, raised blood pressure; $T C+v e$, positive throat culture; $S S+v e$, positive skin culture; $\downarrow C 3$, serum $\mathrm{C} 3$ complement reduced.

*M-types newly associated with PSAGN 
from County Victoria. One isolate was from a skin lesion and three were from the throat.

Non-typable $(T-/ M-/ O F+)$. There were four non-typable Group-A isolates from three female patients, aged 12-13 years, one yielding isolates from skin and throat. All were from County Victoria. These are possibly new strains which did not react with the current panel of typing antisera.

\section{Monthly distribution of streptococcal serotypes}

The epidemic of 1986 appeared to be bimodal. The first phase was in March-May and the second phase July-October. The figure shows the isolation rates of the various serotypes throughout the year. Isolations of M-type 48 preceded the first wave of the epidemic and peaked in February, whereas Mtype 73, PT 5757 and, to a lesser extent, M-type 55, were prevalent during the first phase. Thereafter, isolation of M-types 73 and PT 5757 fluctuated throughout the year while M 55 disappeared in May. M-type 11 isolations peaked just after the first wave, being prevalent in May and June when most other serotypes were relatively inactive and M-type 73 was in decline. PT 180 isolations peaked in August, early in the second phase, while M-type 55 reappeared in August and was prevalent during the late second phase, peaking in September at which time isolates of M-type 73 and PT 5757 were also made.

\section{Discussion}

Nephritogenicity of a group-A streptococcal serotype may be inferred from its frequent association with PSAGN and its relative infrequency in uncomplicated pyoderma. The isolation of a streptococcal strain from the skin of a PSAGN patient was not assumed to be evidence for nephritogenicity. It has been shown that $18 \%$ of children from whom streptococci were recovered from more than one site were found to be carrying two distinct strains ${ }^{9}$ and serial cultures of uncomplicated pyoderma showed changes in streptococcal type between weekly cultures on $28 \%$ of occasions. Infections of single lesions with streptococci of more than one group were reported in children in an urban school ${ }^{10}$ but mixtures of different serotypes of group $\mathrm{A}$ in a single lesion were rare $(1 \%)$.

Throughout 1986, six serotypes of group-A streptococci were recorded in Trinidad. The nephritogenic M-type 55 reappeared in the community in February and, together with M-type 73 and the provisional type (PT) 5757 were isolated frequently from patients with PSAGN during the first phase of the bimodal epidemic, March-May. A retrospective search revealed that during 1966
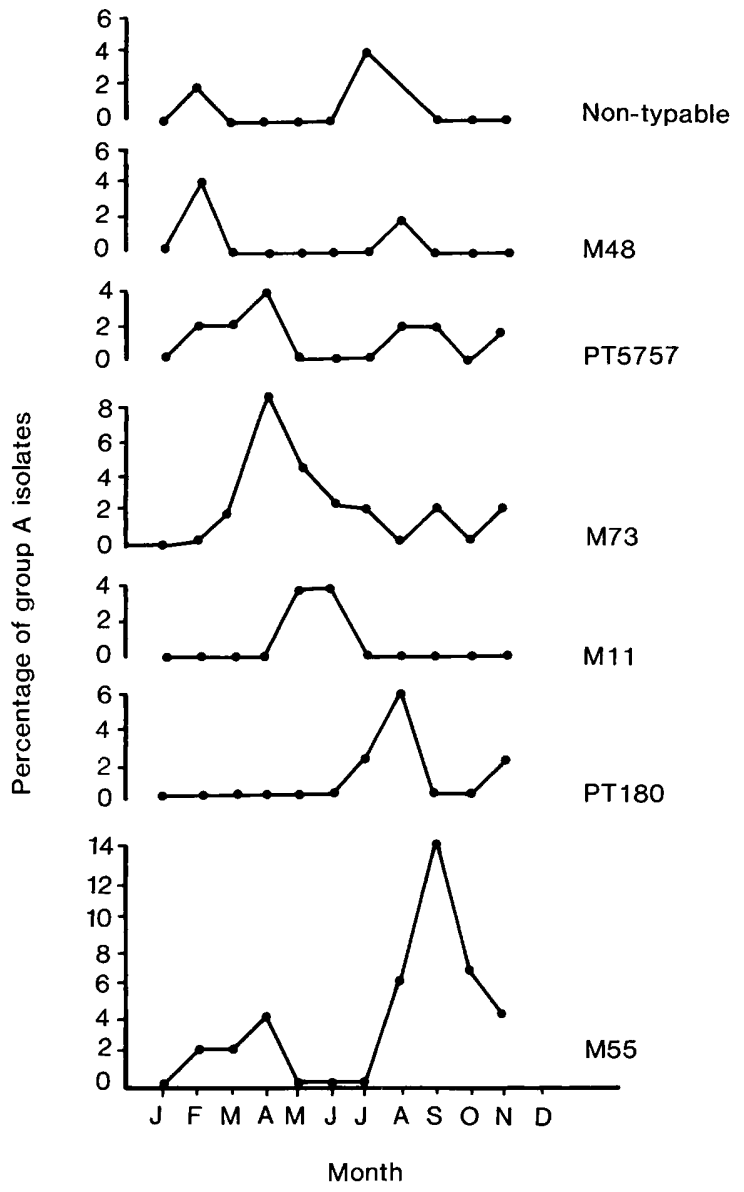

Figure. Monthly distribution of streptococcal serotypes most frequently isolated in Trinidad during 1986 are presented. Note the percentage of non-typable serotypes occurring between the two phases of PSAGN.

and the first half of 1967 there were 42 cases of PSAGN which yielded, on streptococcal T-typing, Type 3/13/B3264 (D.C.J.B., unpublished data). Isolates from 32 of the cases were available for testing and 23 of them gave the same M-type antigen as the provisional type PS346 later designated M-type 73. At that time, this serotype was found to infect $19 \%$ of PSAGN patients from whom positive cultures were obtained. During the epidemic of 1986 , M-type 73 was again found in $21.2 \%$ of the PSAGN patients whose cultures were serotyped. Recently, M-type 73 has been reported to cause persistent streptococcal infection in a primary school in England but none of the children developed PSAGN, perhaps because of prompt penicillin therapy. ${ }^{11}$ Between January 1980 and June 1987, M-type 73 had been associated with one definite diagnosis of PSAGN and may have been implicated in two probable diagnoses. ${ }^{8}$ In this series, M-type 73 was associated with at least 10 
documented PSAGN patients; this number is probably an underestimate.

It was surprising to isolate PT 5757 from PSAGN cases in Trinidad. Previously, such serotypes had been identified only among isolates from Western Europe - in the UK and the Federal Republic of Germany in particular. Between 1980 and 1987, 25 isolates of this type were made from skin lesions of meat workers in the $\mathrm{UK}^{8}$ and from sore throats in hospital patients. First isolated by Dr R. C. Hart (Public Health Laboratory, Exeter) in 1979, these strains have also been isolated during outbreaks of surgical sepsis in West Germany. ${ }^{12}$ Whereas in these countries there are no reports of an association with PSAGN, in Trinidad this type seems to be skin associated and linked with PSAGN, since six of the seven isolates serotyped were cultured from skin lesions of PSAGN patients.

Three isolates of M-type 48 from two PSAGN cases were of great interest. This serotype had not been isolated in Trinidad before nor, as far as can be ascertained, in the West Indies. The Central Public Health Laboratory had recorded only a single previous case involving a schoolboy who developed PSAGN after acquiring pyoderma while overseas.

Provisional type 180 has been isolated from cultures taken from throat swabs of both PSAGN and acute rheumatic fever (ARF) cases in Trinidad over the last 10 years. In 1986, however, there were four isolates from PSAGN patients and only one from an ARF patient. First isolated in 1972 by H. M. Rice (General Hospital, Nottingham), it has been known to cause outbreaks of skin sepsis. ${ }^{12}$

The isolation of M-type 11 from patients with PSAGN were surprising as this strain is usually associated with ARF. The Trinidad M-type 11 is a variant of the classical M-type 11 which was classified by Parker as a nephritogenic strain. ${ }^{13}$ The isolation of this strain from PSAGN patients is a departure from our observations of the last 15 years.

Thus, while the bimodal epidemic of PSAGN in 1986 was partly the result of antecedent streptococcal infection by M-type 73 during the first phase and by the documented nephritogenic M-type 55 during the second phase, there were also contributing serotypes new to Trinidad and Tobago (e.g. Mtype 48 infections preceded the first phase, peaking in February, and PT 5757 was prevalent during the first wave of the epidemic). M-type 11 followed the first wave peaking in May-June, and PT 180 infections which peaked in August preceded the explosive M-type 55 second wave which peaked in September and declined therafter.
The General Hospital, San Fernando, serves the Counties of Victoria, St Patrick, Caroni and Nariva/Mayaro. A clear and significant observation of the geographic distribution was the high streptococcal activity in County Victoria. While 40 isolates (76.92\%) (14 of which were M-type 55) were cultured from PSAGN patients from County Victoria, only $6(11.54 \%)$ were from County St Patrick, $5(9.62 \%)$ from Caroni and $1(1.92 \%)$ from Nariva/Mayaro. The reasons for this skewed distribution are still unclear.

This paper was offered for publication with the permission of the Chief Medical Officer, Ministry of Health, Sackville Street, Port of Spain, Trinidad.

\section{REFERENCES}

1. Poon-King T, Potter E V, Svartman M et al. Epidemic acute nephritis with reappearance of M-type 55 streptococci in Trinidad. Lancet 1973; $1: 475-479$.

2. Poon-King T, Mayon-White R T, Harnaryan C et al. Acute glomerulonephritis and rheumatic fever in South Trinidad, 1975-1978. In: Parker M T (ed) Pathogenic streptococci. Surrey, Reedbooks. 1979: 124-125.

3. Svartman M, Potter E V, Finklea J F, Poon-King T, Earle D P. Epidemic scabies and acute glomerulonephritis in Trinidad. Lancet 1972; 1: 249-251.

4. Reid H F M, Bassett D C J, Poon-King T, Zabriskie J B, Read S E. Group G streptococci in healthy school children and in patients with glomerulonephritis in Trinidad. J Hyg (Lond) 1985; 94:61-68.

5. Gnann J W, Gray B M, Griffin F M, Dismukes W E. Acute glomerulonephritis following group $\mathrm{G}$ streptococcal infection. $J$ Infect Dis 1987; 156: 41 1-412.

6. Reid H F M, Poon-King T. Epidemic scabies and associated acute glomerulonephritis in Trinidad. Bull Pan Am Health Organization 1988; 22, 103-107.

7. Reid H F M, Read S E, Zabriskie J B. Specific blastogenic response to group-A streptococcal cell-wall and cellmembrane antigens in acute glomerulonephritis patients in Trinidad. In: Parker M T (ed) Pathogenic streptococci. Surrey, Reedbooks. 1979: 99-100.

8. Gaworzewska E, Colman G. Changes in the pattern of infection caused by Streptococcus pyogenes. Epidemiol Infect 1988; 100: 257-269.

9. Parker M T. Streptococcal skin infection and acute glomerulonephritis. Br J Dermatol 1969; 81 Suppl 1: 37-46.

10. Bassett D C J. Streptococcal pyoderma and acute nephritis in Trinidad. Br J Dermatol 1972;86 Suppl 8: 55-61

11. Briscoe $J$ H D. Persistent streptococcal throat infection in a preparatory school for boys. J Hyg (Lond) 1985; 95 : 671-676.

12. Fraser C A M, Colman G. Some provisional M-types among Streptococcus pyogenes (Lancefield Group A). In: Kimura Y, Kotami S, Shiokawa Y (eds) Recent advances in streptococci and streptococcal diseases. Bracknell, Berkshire, Reedbooks. 1985: 35.

13. Parker M T, Tomlinson A J H, Williams R E O. Impetigo contagiosa: the association of certain types of Staphylococcus aureus and of Streptococcus pyogenes with superficial skin infections. J Hyg (Lond) 1955; 53 : 458473. 